Supporting information

\section{Effect of microplastic on the gills of the Shore Crab Carcinus maenas}

Andrew J.R. Watts ${ }^{\text {1*+, Mauricio A. Urbina }}{ }^{1,2 \dagger}$, Rhys Goodhead ${ }^{1}$, Julian Moger ${ }^{3}$ Ceri Lewis ", Tamara S. Galloway ${ }^{\text {' }}$

${ }^{1}$ College of Life and Environmental Sciences: Biosciences, Geoffrey Pope Building, University of Exeter, Stocker Road, Exeter EX4 4QD, United Kingdom

${ }^{2}$ Departamento de Zoología, Facultad de Ciencias Naturales y Oceanográficas, Universidad de Concepción, Casilla 160-C, Concepción, 4070386, Chile.

${ }^{3}$ School of Physics, University of Exeter, Exeter, EX4 4QL, United Kingdom

*Corresponding author: *Phone: +44 (0)1392724677 fax: +44 (0) 1392263700 Email: a.watts.research@gmail.com

${ }^{\dagger}$ First Authorship is shared between AJRW and MAU as they equally contributed to this manuscript.

KEYWORDS: Microplastic, polystyrene, oxygen uptake, Ecotoxicology, Marine pollution, salinity, ion regulation, osmoregulation

\section{Content}

Figure SI1 Extra results from the normal salinity experiments

Page S2

Figure SI.2 Extra results from the reduced salinity experiments

Page S3

Figure SI3 Change in $\mathrm{pH}$ to determine buffering capacity

Page S4

Table SI.1 General Linear Model output of experiments 

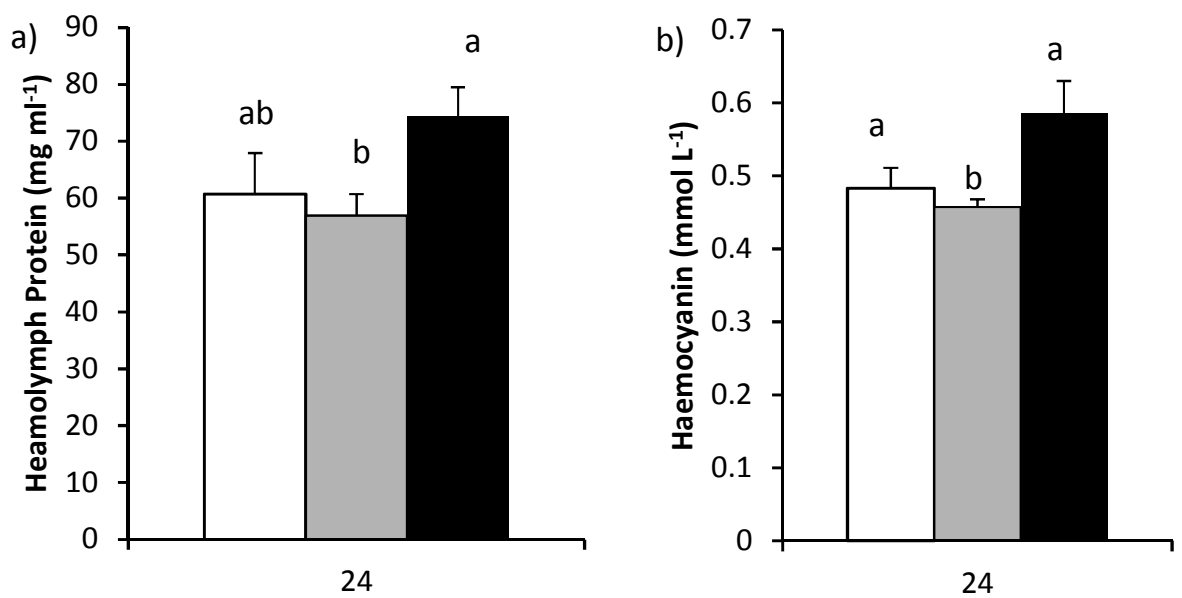

c)

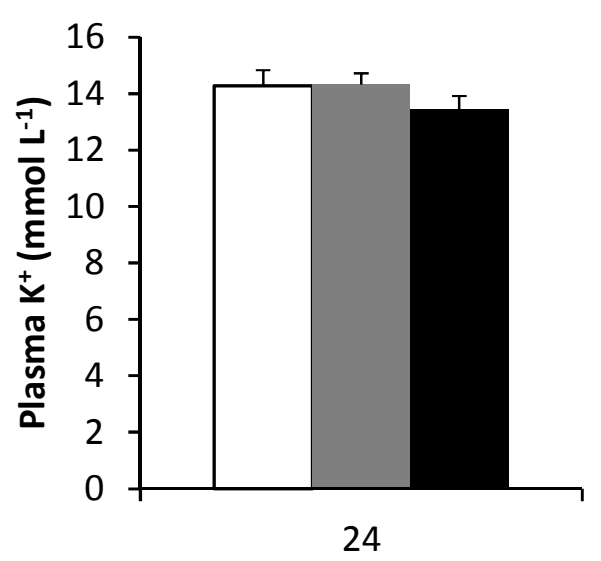

Figure SI1 Extra results from the normal salinity experiments. a) Hemolymph protein concentration, b) heamocycine concentration. C) Plasma $\mathrm{K}^{+}$concentration $24 \mathrm{~h}$ post addition of plastic. In the shore crab Carcinus maenas subjected to three treatments of $8 \mu \mathrm{m}$ microplastic. White bars crabs with no plastic added to the tank $(\mathrm{n}=10)$, grey bars crabs with $10^{6}$ microspheres $\mathrm{L}^{-1}$ within $2 \mathrm{~L}$ of water. Black bars represent crabs with $10^{7}$ microspheres L-1 with 2 L water added. Error bars are one standard error. Means that do not share a letter are significantly different. Bars with no letters indicate no significant difference. 

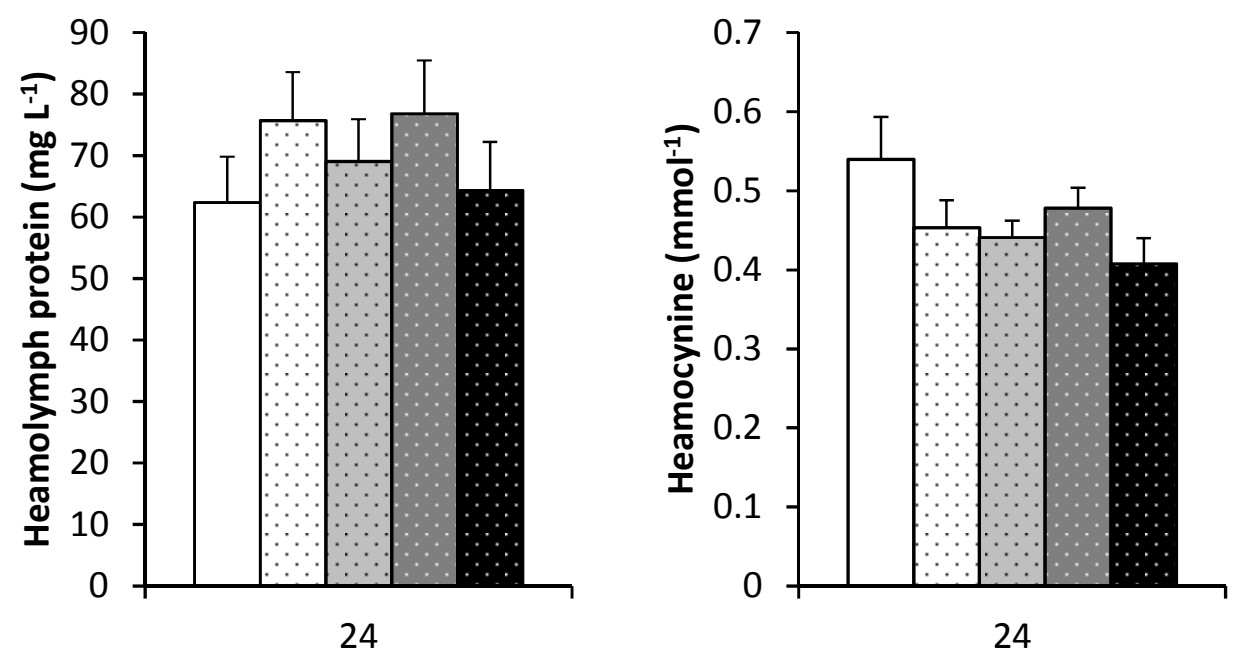

35
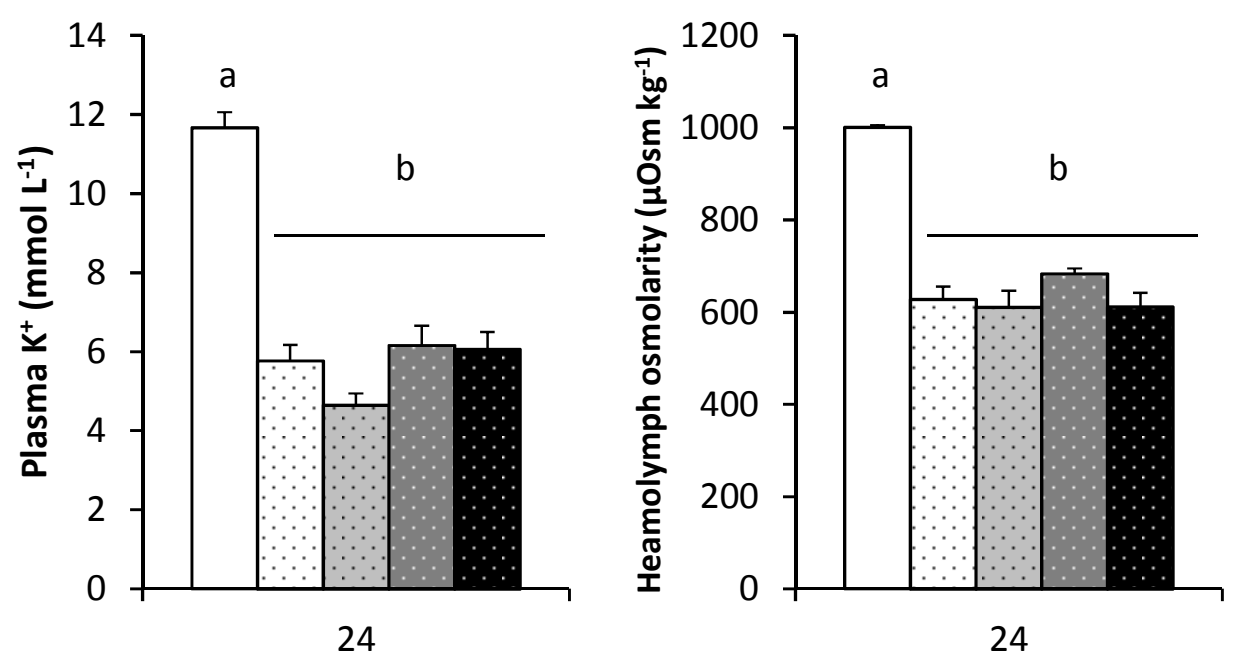

Figure SI.2 Extra results from the reduced salinity experiments. a) Hemolymph protein concentration, b) heamocycine concentration. C) Plastic $\mathrm{K}+$ concentration d) Heamolymph osmolality $24 \mathrm{~h}$ post salinity change. In the shore crab Carcinus maenas subjected to four treatments of $8 \mu \mathrm{m}$ microplastic. White bars crabs with no plastic added to the tank $(n=10)$, light grey bars crabs with $10^{5}$ microspheres $\mathrm{L}^{-1}$ grey bars crabs with $10^{6}$ microspheres $\mathrm{L}^{-1}$ within $2 \mathrm{~L}$ of water. Black bars represent crabs with $10^{7}$ microspheres $\mathrm{L}^{-1}$ with $2 \mathrm{~L}$ water added. Dots within bars represent crabs that have been added to $10 \mathrm{ppt}$ artificial sea water after 16h of plastic exposure. Clear bars represent crabs changed into clean 33ppt ASW. Error bars are one standard error. Means that do not share a letter are significantly different. Bars with no letters indicate no significant difference. Significant differences in oxygen consumption were tested at each time point independent of each other time points. 
67

\section{SI.3 determining the charge on the plastic}

A titration method was used to determine the charge on the neutral, Amino and Carboxyl polystyrene microspheres. To do this a sample of each plastic $\left(1,000\right.$ particles $\left.\mathrm{ml}^{-1}\right)$ was made up in duplicate $20 \mathrm{ml}$ deionised water. Initial $\mathrm{pH}$ was measured in each sample after stripping $\mathrm{CO}_{2}$ by bubbling nitrogen (ca. 3 mins). Once initial pH was stable volumes of between $2-4 \mu 1$ of a $0.0025 \mathrm{M} \mathrm{HCl}$ was added to the samples in a Metrohm autotitrator (815 Robotic USB Sample Proces-201 sor XL, Switzerland) until a $\mathrm{pH}$ of 5.9 was reached. This took approximately $1 \mathrm{~h}$ per sample. $\mathrm{pH}$ was measured in each sample. Accumulative acid and $\mathrm{pH}$ (from initial $\mathrm{pH}$ to around a $\mathrm{pH}$ of 6.4) was then plotted and the inflection point was determined as the middle value from where the buffering capacity was reached ( $\mathrm{pH}$ stared to drop) and where it was saturated (where $\mathrm{pH}$ levels off). The difference between Deionised water (with no plastic) and the water with plastics added was determined.

Amino coated plastics had a lower starting pH (6.969) than DI (7.137) and neutral (7.215) and Carboxyl (7.222). The inflection point of DI water and amino particles were after $18 \mu \mathrm{l}$ acid, while Neutral and Carboxyl inflected after $20 \mu \mathrm{l}$ acid. This shows that the Neutral and Carboxyl have a slight positive charge and the Amino does not. It is suggested Amino has a negative charge; it is suggesting that it has a less positive charge from the Neutral but the level of negative charge could not be determined. That said the charge is very small about a $+5 \mu$ mol of $\mathrm{H}^{+}$ions. Sea water has a buffering capacity of ca. $2000 \mu \mathrm{mol} \mathrm{H}+$ therefore it was not possible to detect any charge in sea water.
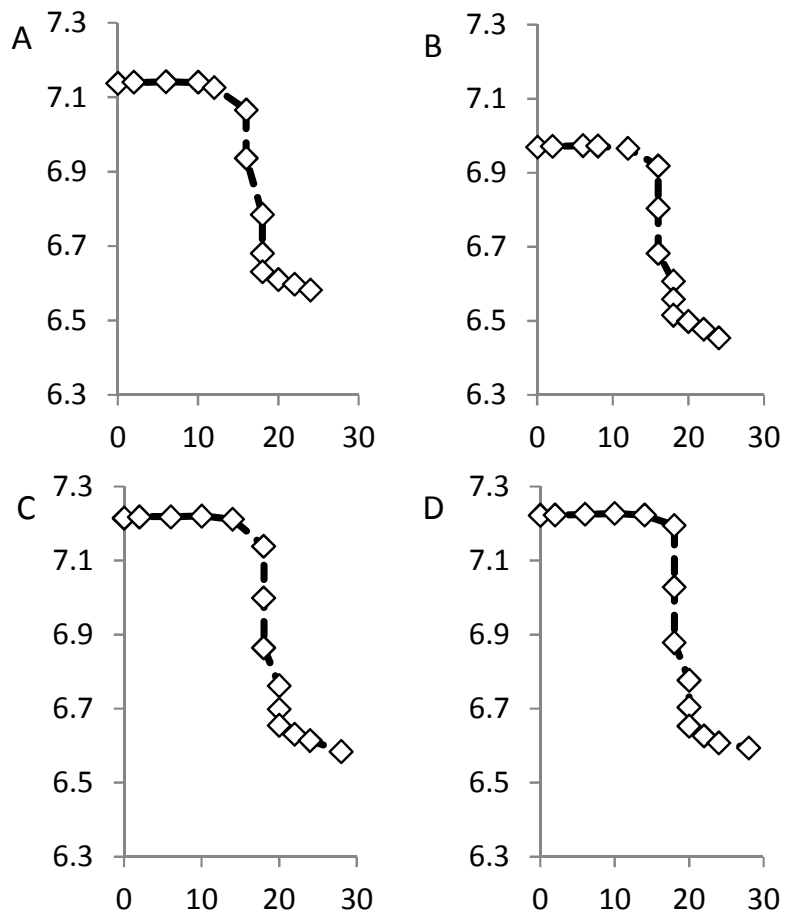

Cumulative amount of Acid $(\mu 1)$

Figure SI3 Change in $\mathrm{pH}$ to determine buffering capacity of DI with A) nothing added, B) aminated $\left(\mathrm{NH}_{2}\right)$ polystyrene added $\mathrm{C}$ ) polystyrene added $\mathrm{D}$ )carboxylated $(\mathrm{COOH})$ polystyrene added 
73 Table SI.1 General Linear Model along with Means \pm SE of measurements of crabs dosed with different types

74 of particles in different surface coatings. (A) Oxygen consumption prior to particles applied (initial) and 1h, 19h

75 and 24 hours post plastic dosing. (b) Heamolymph constituents of Protein, Haemocyanin and ions of $\mathrm{Ca}^{2+}, \mathrm{K}^{+}$

$$
\text { and } \mathrm{Na}^{+}
$$

\begin{tabular}{|c|c|c|c|c|c|c|c|c|c|}
\hline & $\mathrm{F}$ & df & $\begin{array}{r}\text { type } \\
\text { Conc. }\end{array}$ & $\begin{array}{c}\text { Control } \\
0\end{array}$ & $\begin{array}{c}\text { Sediment } \\
10^{6}\end{array}$ & $\begin{array}{c}\text { Carboxyl } \\
10^{6} \\
\end{array}$ & $\begin{array}{c}\text { Aminate } \\
10^{6}\end{array}$ & $\begin{array}{c}\text { Carboxyl } \\
10^{7} \\
\end{array}$ & $\begin{array}{c}\text { Aminate } \\
10^{7} \\
\end{array}$ \\
\hline \multicolumn{10}{|c|}{ (A) Oxygen consumption $\left(\mathrm{ml} \mathrm{O2} \mathrm{g}^{-1} \mathrm{~h}^{-1}\right)$} \\
\hline Initial & 0.75 & 5,55 & 0.587 & $\begin{array}{r}0.0380 \\
\pm 0.0040\end{array}$ & $\begin{array}{r}0.0446 \\
\pm 0.0063\end{array}$ & $\begin{array}{r}0.0365 \\
\pm 0.0040\end{array}$ & $\begin{array}{r}0.0438 \\
\pm 0.0053\end{array}$ & $\begin{array}{r}0.0375 \\
\pm 0.0046\end{array}$ & $\begin{array}{r}0.0425 \\
\pm 0.0069\end{array}$ \\
\hline Post $1 \mathrm{hr}$ & 1.28 & 5,54 & 0.289 & $\begin{array}{r}0.0373 \\
\pm 0.0050\end{array}$ & $\begin{array}{r}0.0525 \\
\pm 0.0066\end{array}$ & $\begin{array}{r}0.0378 \\
\pm 0.0046\end{array}$ & $\begin{array}{r}0.0483 \\
\pm 0.0063\end{array}$ & $\begin{array}{r}0.0370 \\
\pm 0.0060\end{array}$ & $\begin{array}{r}0.0475 \\
\pm 0.0072\end{array}$ \\
\hline Post $19 \mathrm{hr}$ & 0.65 & 5,54 & 0.660 & $\begin{array}{r}0.0326 \\
\pm 0.0048\end{array}$ & $\begin{array}{r}0.0421 \\
\pm 0.0076\end{array}$ & $\begin{array}{r}0.0328 \\
\pm 0.0058\end{array}$ & $\begin{array}{r}0.0426 \\
\pm 0.0061\end{array}$ & $\begin{array}{r}0.0329 \\
\pm 0.0057\end{array}$ & $\begin{array}{r}0.0405 \\
\pm 0.0062\end{array}$ \\
\hline Post $24 \mathrm{hr}$ & 1.39 & 5,53 & 0.245 & $\begin{array}{r}0.0317 \\
\pm 0.0062\end{array}$ & $\begin{array}{r}0.0455 \\
\pm 0.0071\end{array}$ & $\begin{array}{r}0.0428 \\
\pm 0.0064\end{array}$ & $\begin{array}{r}0.0429 \\
\pm 0.0064\end{array}$ & $\begin{array}{r}0.0336 \\
\pm 0.0070\end{array}$ & $\begin{array}{r}0.0393 \\
\pm 0.0056\end{array}$ \\
\hline \multicolumn{10}{|c|}{ (B) Heamolymph constituents } \\
\hline $\begin{array}{l}\text { Protein } \\
\left(\mathrm{mg} \mathrm{ml}^{-1}\right)\end{array}$ & 0.72 & 5,53 & 0.611 & $\begin{array}{r}44.50 \\
\pm 20.79\end{array}$ & $\begin{array}{r}69.54 \\
\pm 14.73\end{array}$ & $\begin{array}{r}64.37 \\
\pm 21.53\end{array}$ & $\begin{array}{l}42.06 \\
\pm 8.53\end{array}$ & $\begin{array}{r}35.02 \\
\pm 14.92\end{array}$ & $\begin{array}{r}69.91 \\
\pm 22.83\end{array}$ \\
\hline $\begin{array}{l}\text { Haemocyanin } \\
\text { (mg g protein) }\end{array}$ & 0.17 & 5,53 & 0.974 & $\begin{array}{r}0.68 \\
\pm 0.10\end{array}$ & $\begin{array}{r}0.75 \\
\pm 0.05\end{array}$ & $\begin{array}{r}0.79 \\
\pm 0.09\end{array}$ & $\begin{array}{r}0.72 \\
\pm 0.08\end{array}$ & $\begin{array}{r}0.71 \\
\pm 0.13\end{array}$ & $\begin{array}{r}0.77 \\
\pm 0.09\end{array}$ \\
\hline $\begin{array}{l}\mathrm{Ca}^{2+} \\
(\mathrm{mM})\end{array}$ & 0.42 & 5,53 & 0.832 & $\begin{array}{l}67.16 \\
\pm 2.28\end{array}$ & $\begin{array}{l}65.68 \\
\pm 1.51\end{array}$ & $\begin{array}{l}66.96 \\
\pm 1.48\end{array}$ & $\begin{array}{l}64.89 \\
\pm 2.01\end{array}$ & $\begin{array}{l}67.40 \\
\pm 1.02\end{array}$ & $\begin{array}{l}64.85 \\
\pm 1.89\end{array}$ \\
\hline $\begin{array}{l}\mathrm{K}^{+} \\
(\mathrm{mM})\end{array}$ & 1.04 & 5,53 & 0.405 & $\begin{array}{l}12.40 \\
\pm 0.47\end{array}$ & $\begin{array}{l}12.51 \\
\pm 0.41\end{array}$ & $\begin{array}{l}12.94 \\
\pm 0.58\end{array}$ & $\begin{array}{l}12.41 \\
\pm 0.37\end{array}$ & $\begin{array}{l}13.37 \\
\pm 0.40\end{array}$ & $\begin{array}{l}14.14 \\
\pm 1.36\end{array}$ \\
\hline $\begin{array}{l}\mathrm{Na}^{+} \\
(\mathrm{mM})\end{array}$ & 1.98 & 5,53 & 0.099 & $\begin{array}{l}550.00 \\
\pm 14.00\end{array}$ & $\begin{array}{l}516.80 \\
\pm 14.00\end{array}$ & $\begin{array}{r}534.47 \\
\pm 8.64\end{array}$ & $\begin{array}{r}525.73 \\
\pm 8.12\end{array}$ & $\begin{array}{l}551.40 \\
\pm 10.29\end{array}$ & $\begin{array}{r}515.23 \\
\pm 8.17\end{array}$ \\
\hline
\end{tabular}

